\title{
Micro-organismes anti-cancéreux et armement
}

> Depuis plusieurs années, la recherche sur les micro-organismes pour une utilisation à des fins d'immunothérapie antitumorale est en plein essor. L'efficacité antitumorale de ces micro-organismes repose sur trois mécanismes principaux : la destruction des cellules tumorales, la stimulation du système immunitaire et la reprogrammation du microenvironnement tumoral. Afin d'optimiser leur action immunothérapeutique, ces micro-organismes peuvent être génétiquement modifiés pour les rendre capables de vectoriser des molécules immunostimulantes ou des anticorps. Par ingénierie moléculaire, il est désormais possible de diversifier les formats et fonctions de ces anticorps afin d'inhiber les points de contrôle immunitaire ou encore de recruter les cellules immunitaires effectrices au site de la tumeur. Cette Synthèse s'intéresse particulièrement à ces innovations et à leurs avantages en immunothérapie. <

En ces temps de pandémie de COVID-19, les mots «virus », «microbe », «pathogène » sont systématiquement associés aux notions de maladie, de danger et de mortalité. Pourtant, depuis plus d'un siècle, certains agents biologiques sont utilisés au service de la santé humaine. Afin de réconcilier ces deux mondes, apparemment en opposition, cette revue s'attache à décrire la stratégie d'immunothérapie oncolytique utilisant virus, bactéries ou protozoaires, qui offre de nouvelles perspectives dans la lutte contre le cancer, un fléau mondial. Bien que cette stratégie soit en plein développement depuis une dizaine d'années, la preuve de concept sur laquelle elle repose a été établie depuis la fin du XIX ${ }^{\mathrm{e}}$ siècle. En effet, c'est au début des années 1890 que le Dr William Coley ${ }^{1}$, un chirurgien assistant et instruc-

Vignette (Photo @ Inserm/Galon, Jérôme).

1 William Coley ne connaissait pas en fait les travaux antérieurs de deux médecins allemands, Karl David Wilhelm Busch et Friedrich Fehleisen, tombés quelque peu dans l'oubli. En 1868, Busch fût le premier à inoculer intentionnellement un patient cancéreux avec un bouillon bactérien isolé à partir d'un érysipèle [1] tandis que Fehleisen répéta l'expérience en 1882 chez cinq patients : il identifia alors l'agent causal de l'érysipèle comme étant la bactérie Streptococcus pyogenes [2].

\section{Le couteau suisse}

\section{de I'immunothérapie}

Loïs Coënon, Arthur Battistoni, Agathe Poupée-Beaugé, Stéphanie Germon, Isabelle Dimier-Poisson

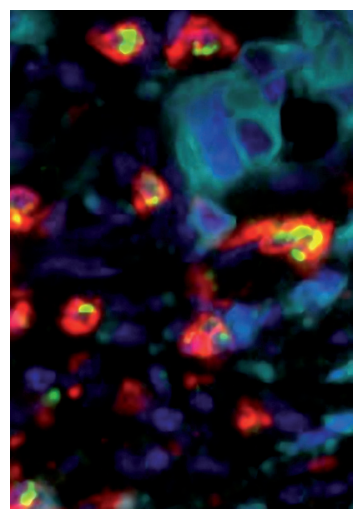

Équipe BioMAP,

Université de Tours, INRAe, 31 avenue Monge, 37200 Tours, France.

dimier@univ-tours.fr

teur en chirurgie à l'école de médecine de New York, rapporte qu'il a traité avec succès des patients atteints de sarcome par injection locale de la bactérie Streptoccocus pyogenes, après avoir constaté la guérison d'un patient cancéreux à la suite d'un érysipèle, une infection cutanée [3-5]. Il met ainsi en évidence une corrélation entre la survenue d'une infection aiguë et la régression spontanée d'une tumeur cancéreuse. Malheureusement, ses observations ne suscitent plus l'intérêt des chercheurs à la suite de l'avènement, au $x x^{e}$ siècle, de la chimiothérapie et de la radiothérapie. Ses travaux vont tomber dans l'oubli jusqu'au début des années 1950. Il faudra alors les travaux d'un autre new-yorkais, Lloyd Old [6], pour que l'idée que l'utilisation de bactéries (en l'occurrence le bacille Calmette et Guérin, BCG) conduit à des réponses anti-tumorales, soit concrétisée. Puis, en 1969, Georges Mathé et ses collègues publient des résultats prometteurs sur le traitement de leucémies lymphoblastiques par le BCG [7] mais ceux-ci ne sont pas reproduits par d'autres chercheurs. II faudra attendre 1976 pour qu'Alvaro Moralès et ses collègues démontrent, chez des patients souffrant de tumeurs superficielles de la vessie, l'efficacité du BCG [8], dont l'utilisation est encore aujourd'hui recommandée pour les tumeurs de la vessie à haut risque de récidive et de progression [9]. Autour de ces mêmes années 1970, il avait été également observé qu'une infection chronique par le protozoaire intracellulaire Toxoplasma gondii, voire l'administration d'extraits de ce même protozoaire conférait une résistance, chez la souris, à de nombreux pathogènes incluant des virus [10], des bactéries [11], des parasites [12], des levures [13], ainsi également qu'à des tumeurs mammaires 
[14]. Les progrès de la biologie moléculaire, de l'ingénierie génétique et de l'immunologie ont désormais fait revenir sur le devant de la scène l'utilisation de micro-organismes modifiés, principalement des virus, comme traitements d'immunothérapie anti-cancéreuse $[38](\rightarrow)$. Ainsi, en 2005 , le premier virus oncolytique, $(\rightarrow)$ Voir la Synthèse $n^{\circ} 2$, février 2013, de J. Pol et al., $m / s$ page 165 l'adénovirus $\mathrm{HlOl}$ (Oncorine ${ }^{\circledR}$ ), reçoit une autorisation d'utilisation en combinaison avec de la chimiothérapie par la State Food and Drug Administration (SFDA) ${ }^{2}$ en République populaire de Chine pour le traitement des cancers de la tête et du cou [15]. En 2015, la Food and Drug Administration (FDA) américaine approuve un herpès virus de type 1 (HSV-1) atténué sécrétant du granulocyte-monocyte colonystimulating factor (GM-CSF) humain (talimogene laherparepvec, Imlygic $\left.{ }^{\circledR}\right)$ comme première monothérapie virale oncolytique pour le traitement local de patients atteints de mélanomes non résécables récidivant après chirurgie initiale [16]. Plus récemment, en 2018, un vaccin fondé sur l'utilisation de la bactérie Listeria monocytogenes (AT-014) atténuée a obtenu de l'United States Department of Agriculture (USDA) - Center for Veterinary Biologics une autorisation conditionnelle d'utilisation pour traiter l'ostéosarcome canin, élargissant ainsi l'arsenal des microorganismes oncolytiques aux bactéries.

Depuis plusieurs années, cet arsenal thérapeutique contre le cancer est renforcé par une nouvelle génération d'agents immunothérapeutiques armés capables d'acheminer soit des molécules cytotoxiques soit de sécréter ou d'exprimer des antigènes tumoraux ou des anticorps afin d'améliorer l'activité oncolytique et la spécificité tumorale. Cette revue présente les mécanismes d'action de ces micro-organismes oncolytiques, les différentes améliorations de leur efficacité thérapeutique dont certaines sont décrites en détail dans la revue de Pol et al. [36], et, pour terminer, la nouvelle génération «tout en un » permettant d'associer en un seul micro-organisme plusieurs stratégies d'immunothérapie.

\section{Mécanismes d'action des micro-organismes oncolytiques}

Certains micro-organismes, comme les protozoaires intracellulaires ou les virus oncolytiques entraînent la destruction des cellules cancéreuses par le simple fait de leur multiplication intracellulaire favorisée par certaines propriétés intrinsèques des cellules tumorales comme la surexpression de récepteurs d'entrée de virus à leur surface, leur prolifération rapide ou encore la dérégulation de certaines voies de signalisation $[6,7,17,18]$. D'autres micro-organismes, comme certaines bactéries, notamment Salmonella enterica serovar Typhimurium (S. Typhimurium), induisent, du fait de leur accumulation dans les cellules tumorales, l'apoptose et l'autophagie cellulaires [19, 20].

Cette activité oncolytique induit également des réponses immunitaires antitumorales innées et adaptatives via la libération de signaux de danger (danger-associated molecular patterns [DAMP]), de pathogen-associated molecular patterns [PAMP], d'antigènes microbiens et tumoraux) dans le microenvironnement tumoral (MET)

\footnotetext{
2 Appelée désormais la National Medical Products Administration (NMPA).
}

immunosuppressif, ayant pour conséquence de briser la tolérance établie et d'augmenter le pouvoir immunogénique de la tumeur [21] (Figure 1).

\section{Manipulations génétiques « anciennes 》 et associations thérapeutiques}

Diverses stratégies génétiques ont été développées afin d'accroître la spécificité et la sélectivité tumorale, le potentiel oncolytique, l'induction d'une immunité antitumorale, tout en limitant la virulence des microorganismes oncolytiques. Pour une description de ces différentes stratégies, nous référons le lecteur à la revue de Pol et al. [38]. À titre d'exemple, on peut citer le PVSRIPO développé pour traiter le glioblastome par injections intracrâniennes. Le PVSRIPO est apparenté au Poliovirus Sabin recombinant dont la partie du génome impliqué dans la neurovirulence, l'internal ribosome entry site (IRES), a été substituée et remplacée par celle du rhinovirus humain de type 2. Le virus conserve ainsi son tropisme naturel pour le récepteur nectin-like molecule 5 [39] $(\rightarrow$ ) (ou CD155), exprimé à la surface des cellules neuronales

\section{$\rightarrow$ Voir la Synthèse} de V. Catros et al., $m / s n^{\circ} 5$, mai 2014, page 537 saines mais surexprimé par les cellules des glioblastomes, tout en étant dépourvu de neurovirulence grâce à la substitution d'IRES.

Un essai clinique de phase I pour le traitement de patients présentant des glioblastomes récurrents (grade IV OMS) (NCT01491893) a montré que le taux de survie parmi les patients ayant reçu une immunothérapie PVSRIPO est plus élevé à 24 et 36 mois post-traitement que celui observé chez les témoins; deux patients étaient encore en vie plus de 69 mois après le traitement [22]. La FDA a accordé la désignation de thérapie de rupture (breakthrough-therapy) du glioblastome au PVSRIPO en mai 2016.

En partant du constat que l'immunité anti-infectieuse pouvait conduire à l'élimination plus ou moins rapide du micro-organisme oncolytique et que l'immunité antitumorale pouvait s'avérer insuffisante pour éliminer toutes les cellules cancéreuses et empêcher le développement de métastases, différentes équipes ont alors armé les micro-organismes oncolytiques pour leur faire exprimer des molécules immunostimulatrices telles que le GM-CSF, des interleukines comme l'interleukine 15 (IL-15) [23] ou des antigènes tumoraux. Cet armement a montré son efficacité dans de nombreuses études. Ainsi, Trypanosoma cruzi, agent étiologique de la maladie de Chagas et inducteur puissant de réponses immunitaires de type Thl et de lymphocytes $T$ CD $8^{+}$persistant à vie dans les tissus de l'hôte infesté, s'est révélé très efficace comme vecteur de délivrance d'antigène tumoral. Une souche de T. cruzi 


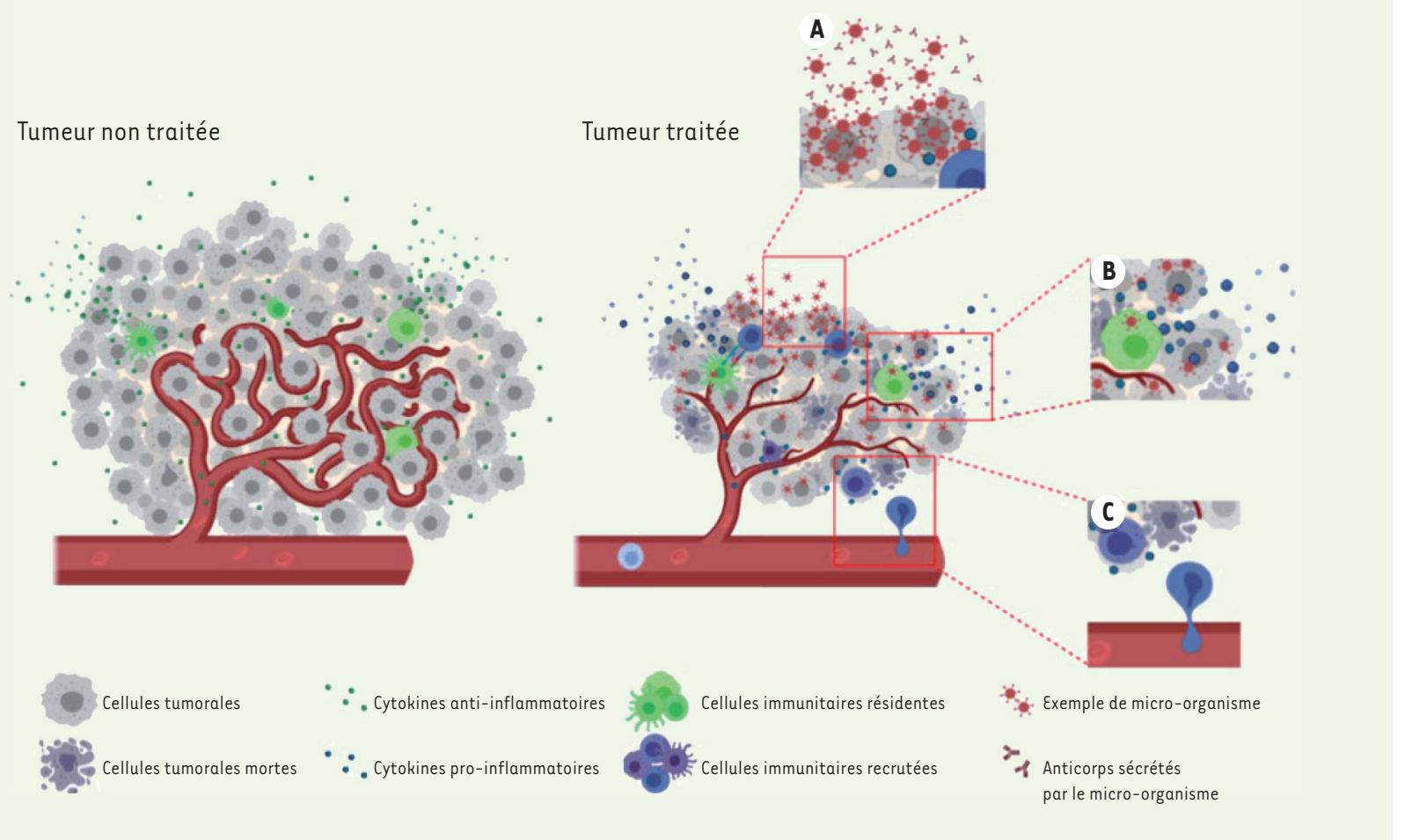

Figure 1. Mécanismes d'action des micro-organismes. Le traitement de tumeurs par des micro-organismes repose sur trois mécanismes principaux. (A) Les micro-organismes infectent les cellules tumorales et, par multiplication intracellulaire ou par accumulation, entraînent la destruction de la cellule tumorale hôte. Afin d'augmenter ce potentiel oncolytique, certains micro-organismes sont génétiquement modifiés pour sécréter des molécules immunomodulatrices, des antigènes tumoraux ou des anticorps thérapeutiques qui seront libérés lors de la destruction de la cellule tumorale. (B) Cette oncolyse directe induit aussi la libération de signaux de dangers dans le microenvironnement tumoral (MET), permettant de lever l'immunosuppression existant dans ce MET. (C) La présence du micro-organisme et des signaux de danger dans la tumeur stimule le système immunitaire inné et adaptatif, induit le recrutement de cellules immunitaires au sein de la tumeur et ainsi potentialise la réponse antitumorale.

atténuée exprimant l'antigène humain NY-ESO-1 (CL14-NY-ESO-1) (un antigène dont l'expression est normalement restreinte aux cellules germinales des testicules adultes et des ovaires fœtaux mais fréquente sur les cellules tumorales), a montré qu'elle induisait des réponses cellulaires T spécifiques de cet antigène humain et conférait une protection solide contre le développement du mélanome et de ses formes métastatiques, dans un modèle murin [24].

De plus, diverses études ont montré un bénéfice dans l'association de micro-organismes armés avec la chimiothérapie pour limiter la toxicité générale des cytotoxiques. Trois essais cliniques de phase I/ II effectués avec des patients atteints de glioblastomes, de cancers colorectaux métastatiques ou avancés sont en cours de recrutement (respectivement NCT03294486, NCT04194034 et NCT03724071) pour tester le TG6002 (Transgene, IIlkirch-Graffenstaden, France). Cet agent est un virus de la vaccine exprimant une protéine hétérologue, la cytosine déaminase $(C D)$, permettant de convertir la prodrogue 5 -fluorocytosine $(5-F C)$ en 5 -fluorouracil $(5-F U)$, chimiothérapie cliniquement approuvée [25]. Sur le même principe, la souche atténuée de $S$. Typhimurium, VNP20009, a été modifiée pour exprimer la CD (souche TAPET-CD). Une étude clinique a montré une colonisation de la tumeur par TAPET-CD associée à une forte production de 5-FU dans cette dernière par rapport aux tissus sains, malgré une colonisation tumorale moins importante que dans les modèles murins testés [26].

Dans le but de traiter le cancer colorectal (CRC), une souche d'Escherichia coli ( $\varepsilon d a-I 1-H I p A)$ a été générée pour arborer à sa surface une protéine de Streptococcus gallolytocus, l'histone-like protein A (HIpA) [27]. Cette protéine interagit avec les héparanes sulfates particuliers des cellules du CRC et permet à la bactérie recombinante de ne persister qu'au niveau de cellesci après administration par voie orale. De plus, cette bactérie exprime la myrosinase, enzyme dégradant les glucosinolates, des molécules retrouvées dans les légumes crucifères, en sulforaphane, composé cytotoxique pour les cellules cancéreuses. Ainsi, les bactéries de la souche $\varepsilon d a-\mid l-H I p A$, après infection orale associée à un régime de légumes crucifères (comme le chou-fleur, le brocoli, le radis...), induisent une diminution du nombre et de la taille des tumeurs dans un modèle murin de CRC, avant d'être éliminées par voie naturelle. 


\section{La solution «tout en un 》}

Parallèlement au développement des virus oncolytiques, d'autres stratégies d'immunothérapie ont vu le jour. L'utilisation d'anticorps monoclonaux thérapeutiques inhibiteurs des points de contrôle immunitaire (immune checkpoints inhibitors, ICI) comme PD-1 (programmed cell death 1), PD-Ll (programmed cell death-ligand 1) ou CTLA-4 (cytotoxic T-lymphocyte-associated protein 4) a révolutionné le traitement de certains cancers, mais pour un nombre limité de patients (20-25\%), tout en étant très onéreuse et s'accompagnant d'une toxicité parfois importante, notamment immunologique. Le développement de micro-organismes oncolytiques multifonctionnels capables de sécréter ces biomédicaments au sein de la tumeur permet non seulement de diminuer les coûts de production mais surtout d'accroître leurs effets thérapeutiques et de démultiplier l'efficacité antitumorale associée à une toxicité plus limitée. Ainsi, le traitement avec CL-14-NY-ESO-1 (voir ci-dessus) associé à un anticorps anti-CTLA-4 s'est révélé efficace pour contrôler le développement d'un mélanome établi [28]. En effet, le blocage de CTLA-4 pendant le traitement augmente la fréquence des lymphocytes T CD8 ${ }^{+}$spécifiques de NY-ESO-l produisant de l'interféron- $\gamma($ IFN $-\gamma$ ) et favorise la migration des lymphocytes vers le microenvironnement tumoral (tumor-infiltrating lymphocytes, TIL). Le traitement du mésothéliome pleural malin par CRS-207, une souche atténuée de Listeria monocytogenes grâce à la délétion de deux gènes et exprimant la mésothéline comme antigène tumoral, en combinaison avec de la chimiothérapie a montré une activité antitumorale encourageante avec un taux de réponse de $59 \%$, ainsi que le recrutement de TIL [29]. Plusieurs essais cliniques sont en cours avec la souche CRS-207 : en association avec le pembrolizumab (un anticorps monoclonal humanisé anti-PD-1, Keytruda ${ }^{\circledR}$ ) pour traiter les cancers gastriques (NCT03122548) ou le mésothéliome pleural (NCT03175172), et avec l'ipilimumab (un anticorps humain anti-CTLA-4, Yervoy ${ }^{\circledR}$ ) (NCT03190265) pour traiter le cancer du pancréas métastatique.

L'écrasante majorité des anticorps thérapeutiques ayant reçu une autorisation de mise sur le marché sont des anticorps entiers de classe $\lg G[30]$. Ce format natif se caractérise par une longue demi-vie grâce au recyclage par le récepteur néonatal pour la région Fc (des IgG) (FcRn) exprimé par les cellules endothéliales vasculaires, ainsi que par l'engagement des RFc $\gamma$ des cellules du système immunitaire, grâce à la présence de cette région Fc. Toutefois, la pénétration de l'anticorps entier dans les tumeurs et à travers les barrières biologiques est faible étant donné sa grande masse moléculaire (environ $150 \mathrm{kDa}$ ) et l'engagement des cellules immunitaires effectrices via les RFc $\gamma$ peut induire des effets indésirables importants. Grâce à l'essor de l'ingénierie moléculaire, d'autres formats d'anticorps plus petits ont été mis au point (Figure 2). Ces fragments d'anticorps ne possèdent pas de région Fc et sont de ce fait plus petits que le format classique, modifiant de façon conséquente leurs caractéristiques. Parmi ces formats, on peut citer les formats fragment antigen binding (Fab) et single-chain fragment variable (scFv) ainsi que les formats découlant de ce dernier, comme les diabodies bispécifiques et les BiTE (bispecific T-cell engagers). Enfin, le plus petit format d'anticorps existant est dérivé des
IgG des camélidés, et est dénommé VHH ou nanobody. Les avantages et inconvénients des différents formats de fragments d'anticorps sont discutés dans la Synthèse de Kitten et al. [40] $(\rightarrow)$.

Diverses études s'intéressent donc au développement de micro-organismes exprimant et/ou sécrétant ces molécules, assurant ainsi la synergie de multiples stratégies d'immunothérapie. Les travaux de Kleinpeter et de ses collègues [31] ont montré la faisabilité d'une telle stratégie en faisant exprimer par des virus recombinants de la vaccine différents formats (anticorps entier, Fab, scFv) de l'IgG J43 dirigée contre la protéine murine PD-1. L'injection intra-tumorale de ces virus armés a montré, dans le modèle tumoral de fibrosarcome MCA 205, une efficacité antitumorale similaire à celle du virus non armé mais combiné à l'administration par voie générale du J43.

En exploitant le mécanisme de ciblage tumoral intrinsèque aux bactéries anaérobies qui survivent dans les régions pauvres en oxygène comme les tumeurs, une autre équipe a développé une souche d'E. coli présentant un mécanisme de lyse dépendant de la densité bactérienne, le quorum-lysis, et produisant en intracellulaire un nanobody ciblant la molécule CD47 (nbCD47), un récepteur souvent surexprimé par les cellules tumorales, qui inhibe la phagocytose (un mécanisme connu sous le nom du «don't eat me signal »). L'accumulation de l'acylhomosérine lors de la multiplication des bactéries de cette souche induit la production d'une protéine de bactériophage, $\varphi \times 174 \varepsilon$, entraînant la lyse de ces bactéries et la libération des molécules nbCD47 directement au site de la tumeur. L'utilisation de cette bactérie recombinante in vitro induit une augmentation de l'activité de phagocytose et, in vivo, une régression tumorale après injection intra-tumorale ou par voie intraveineuse de cellules de lignées cancéreuses surexprimant CD47 [32].

Par ailleurs, pour les virus de grande plasticité génomique comme les poxvirus, il est aussi possible d'obtenir un virus oncolytique exprimant plusieurs molécules hétérologues comme par exemple une cytokine et un inhibiteur de point de contrôle immunitaire. C'est le cas du VV-iPDLl/GM, un virus de la vaccine qui coexprime un inhibiteur de PD-Ll (une protéine de fusion soluble constituée du domaine extracellulaire de PD-1 fusionné à la région $F c$ d'une IgGl humaine) ainsi que le GM-CSF. Ce virus recombinant, dans le modèle murin de mélanome Bl6Fl0, a permis d'obtenir une forte activité antitumorale ainsi que l'induction d'une réponse $T$ cytotoxique spécifique [33]. 


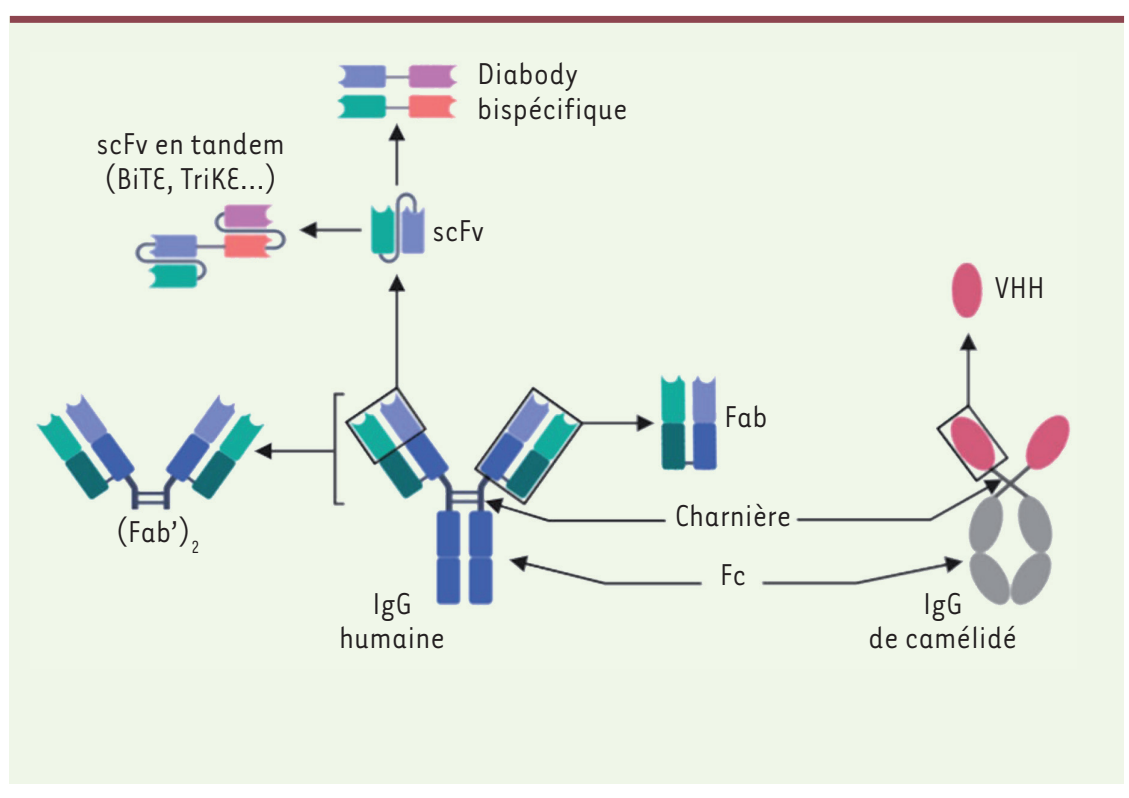

Figure 2. Différents formats de fragments d'anticorps. Le clivage de la région charnière permet d'obtenir les fragments Fab (fragment antigen binding) et $\mathrm{F}(\mathrm{ab})^{\prime}{ }_{2}$. Le fragment scFv (single-chain fragment variable) est obtenu en conservant uniquement les domaines variables, reliés entre eux par un lien peptidique flexible. Des formats dérivés, bispécifiques, comme les diabodies et les scFv en tandem peuvent être également générés. Les BiTE (bispecific T cells engagers) en sont des exemples récents. Enfin, la plus petite sous-unité d'anticorps conservant une capacité de liaison à un antigène est le nanobody (ou VHH pour variable heavy homodimers) provenant d'IgG (immunoglobulines $\mathrm{G}$ ) particulières des camélidés.

Afin d'augmenter la destruction des cellules tumorales par les lymphocytes $T$, il est possible d'utiliser une protéine recombinante bispécifique composée de deux scFv liés par un espaceur, l'un ciblant la molécule CD3 des lymphocytes $T$ et l'autre, un antigène membranaire exprimé par les cellules tumorales. Ce format a été appelé BiTદ. La lyse des cellules tumorales est ainsi induite par la fixation simultanée du BiTE sur les cellules tumorales et sur les lymphocytes T [34]. Le blinatumomab (anti-CD19/anti-CD3, Blincyto ${ }^{\circledR}$ ) est le seul BiTE approuvé par la FDA et l'Agence européenne des médicaments (AEM) pour le traitement de tumeurs malignes de lymphocytes $B$. II est notamment indiqué pour le traitement en monothérapie des enfants, à partir de l'âge de 1 an, ayant une leucémie aiguë lymphoblastique ( $L A L)$ à précurseurs B exprimant CD19 avec chromosome Philadelphie négatif, réfractaire ou en rechute après au moins deux traitements antérieurs, ou en rechute après une allogreffe de cellules souches hématopoïétiques antérieure.

D'autres BiTદ sont en cours de développement et même des TriTદ (trispecific $T$-cell engagers) reconnaissant trois cibles. Certains micro-organismes comme le virus de la vaccine ou un adénovirus ont été modifiés pour exprimer ces BiTદ. L'adénovirus armé pour exprimer un BiTE liant CD3 et le récepteur pour le facteur de croissance épithélial (epidermal growth factor receptor, EGFR) a montré une efficacité tumorale supérieure dans un modèle de xénogreffes grâce à un recrutement et une persistance de lymphocytes T dans la tumeur [35]. II a également été montré récemment que cet adénovirus armé pouvait être délivré dans le microenvironnement tumoral par des cellules souches mésenchymateuses [36]. Enfin, cette stratégie a été adaptée au recrutement de cellules NK, amenant le développement de BiKE (bispecific killer engagers) ainsi que de Trike (trispecific killer engagers). Parmi ces derniers, on peut citer le Trike 161533, développé pour traiter les leucémies myéloïdes aiguës, et qui reconnait à la fois CDI6a (le RFcyllla) exprimé par les cellules NK et CD33 exprimé par les cellules myéloïdes normales et cancéreuses. Cette molécule Trike contient également I'IL-15, une cytokine qui permet la multiplication, l'activation et la survie des cellules NK via son récepteur exprimé par ces cellules [37].

Les micro-organismes oncolytiques sont des outils puissants pour combattre le cancer. Capables de détruire les tumeurs par différents mécanismes, ils sont aussi facilement modifiables grâce à l'évolution de l'ingénierie génétique, afin de mutualiser différentes stratégies d'immunothérapie. Ainsi, la synthèse de molécules de ciblage tumoral ou d'anticorps thérapeutiques par ces micro-organismes permet un meilleur ciblage de la tumeur ainsi qu'une délivrance de molécules thérapeutiques au cœur de celle-ci, engendrant une diminution de la toxicité générale qui leur est associée. Par ailleurs, l'intérêt de ces stratégies thérapeutiques «tout en un » est également économique, puisque le coût des immunothérapies administrées par voie générale est très élevé. Enfin, en sélectionnant le micro-organisme selon son tropisme naturel, il sera possible d'atteindre des tumeurs non accessibles par les techniques chirurgicales, comme certaines tumeurs cérébrales. L'utilisation des microorganismes oncolytiques est donc en plein essor et de nombreuses études pour améliorer leur efficacité en optimisant plusieurs stratégies immunothérapeutiques sont actuellement entreprises.

Compte tenu de leur efficacité thérapeutique potentialisée par une ingénierie génétique adéquate, les micro-organismes modifiés représentent aujourd'hui une étape importante et prometteuse dans la recherche contre le cancer. $\diamond$ 


\section{SUMMARY}

Antitumoral microorganisms: The Swiss army knife of

immunotherapy

Research on viruses, bacteria and protozoa-based immunotherapy has been on the rise for several years. The antitumoral efficacy of these microorganisms relies on three main mechanisms: Destruction of tumor cells, stimulation of the immune response and reprogramming of the tumor microenvironment. In order to optimize their immunotherapeutic action, these microorganisms can be genetically engineered to enhance their tumor-targeting efficacy or to vectorize immunostimulating molecules and/or antibodies. To this aim, molecular engineering allows the design of new antibody formats optimizing their functions. From whole antibodies to tandem single-chain variable fragments, various antibody formats can be vectorized by microorganisms to target receptors such as immune checkpoints or recruit immune effector cells within the tumor. Such possibilities broaden the arsenal of immunotherapeutic cancer treatment. This review focuses on these innovations and their advantages for immunotherapy. $\diamond$

\section{LIENS D'INTÉRÊT}

Les auteurs déclarent n'avoir aucun lien d'intérêt concernant les données publiées dans cet article.

\section{RéFÉRENCES}

1. OBusch W. Aus der Sitzung der medicinischen section vom 13 november 1867. Berlin Klin Wochenschr $1868 ; 5: 137$.

2. Fehleisen $F$. Ueber die züchtung der erysipelkokken auf künstlichem nährboden und ihre übertragbarkeit auf den menschen. Dtsch Med Wochenschr $1882 ; 8$ : 553-4.

3. Coley WB. The treatment of malignant tumors by repeated inoculations of erysipelas. with a report of ten original cases. Am J Med Sci $1893 ; 105: 487-511$.

4. Coley WB. Late results of the treatment of inoperable sarcoma by the mixed toxins of erysipelas and bacillus prodigiosus. Trans Southern Surg Gynecol Ass 1906; $18: 197$.

5. Coley WB. The treatment of inoperable sarcoma by bacterial toxins (the mixed toxins of the Streptococcus erysipelas and the Bacillus prodigiosus). Proc R Soc Med 1910 ; 3 (Surg Sect) : $1-48$.

6. Old LJ, Clark DA, Benacerraf B. Effect of bacillus Calmette Guérin infection on transplanted tumors in the mouse. Nature $1959 ; 184: 291-2$.

7. Mathé G, Amiel JL, Schwarzenberg L, et al. Active immunotherapy for acute lymphoblastic leukaemia. Lancet $1969 ; 1: 697-9$.

8. Morales A, Eidinger D, Bruce AW. Intracavity bacillus Calmette-Guérin in the treatment of superficial bladder tumors. J Urol 1976; 116: 180-3.

9. Babjuk M. European association of urology guidelines on non muscle-invasive bladder cancer (Tatl and carcinoma in situ) - 2019 update. Eur Urology 2019 ; 76 : 639-57.

10. Remington JS, Merigan TC. Resistance to virus challenge in mice infected with protozoa or bacteria. Proc Soc Exp Biol Med 1969 ; 131 : 1184-8.

11. Ruskin J, Remington JS. Immunity and intracellular infection: resistance to bacteria in mice infected with a protozoan. Science $1968 ; 160: 72-4$.

12. Mahmoud AA, Warren KS, Strickland GT. Acquired resistance to infection with Schistosoma mansoni induced by Toxoplasma gondii. Nature 1976 ; $263: 56-7$.

13. Gentry LO, Remington JS. Resistance against Cryptococcus conferred by intracellular bacteria and protozoa. J Infect Dis $1971 ; 123: 22-31$.

14. Hibbs JB Jr, Lambert LH Jr, Remington JS. Resistance to murine tumors conferred by chronic infection with intracellular protozoa, Toxoplasma gondii and Besnoitia jellisoni.J Infect Dis 1971; $124: 587-92$.

15. Liang M. Oncorine, the world first oncolytic virus medicine and its update in China, Curr Cancer Drug Targets 2018 ; $18: 171-6$.

16. Pol J, Kroemer G, Galluzzi L. First oncolytic virus approved for melanoma immunotherapy, Oncoimmunol $2016 ; 5$ : el115641.

17. Lan Q, Xia S, Wang Q, et al. Development of oncolytic virotherapy: from genetic modification to combination therapy. Front Med $2020 ; 14: 160-84$.

18. Feuer $\mathrm{R}$, Whitton JL. Preferential coxsackievirus replication in proliferating/activated cells: implications for virus tropism, persistence, and pathogenesis. Curr Top Microbiol Immunol 2008 ; $323: 149-73$.
19. Sedighi M, Zahedi Bialvaei A, Hamblin MR, et al. Therapeutic bacteria to combat cancer ; current advances, challenges, and opportunities. Cancer Med $2019 ; 8: 3167-81$.

20. Liang K, Liu Q, Li P, et al. Genetically engineered Salmonella typhimurium: recent advances in cancer therapy. Cancer Lett $2019 ; 448: 168-81$.

21. Kuol N, Stojanovska L, Nurgali K, Apostolopoulos V. The mechanisms tumor cells utilize to evade the host's immune system. Maturitas $2017 ; 105: 8-15$.

22. Desjardins A, Gromeier $M$, Herndon $J \varepsilon 2^{\text {nd }}$, et al. Recurrent glioblastoma treated with recombinant poliovirus. $N$ Engl J Med 2018 ; 379 : 150-61.

23. Chaurasiya $S$, Fong $Y$, Warner SG. Optimizing oncolytic viral design to enhance antitumor efficacy: progress and challenges. Cancers (Basel) 2020 ; $12: 1699$.

24. Junqueira C, Santos LI, Galvão-Filho B, et al. Trypanosoma cruzi as an effective cancer antigen delivery vector. Proc Natl Acad Sci USA 2011 ; 108 : 19695-700.

25. Foloppe J, Kempf J, Futin N, et al. The Enhanced tumor specificity of $\operatorname{tg} 6002$, an armed oncolytic vaccinia virus deleted in two genes involved in nucleotide metabolism. Mol Ther Oncolytics 2019; 14 : 1-14.

26. Liang K, Liu Q, Li P, Luo H, Wang H, Kong $Q$. Genetically engineered Salmonella typhimurium: recent advances in cancer therapy. Cancer Lett $2019 ; 448: 168-81$.

27. Ho CL, Tan HQ, Chua KJ, et al. Engineered commensal microbes for dietmediated colorectal-cancer chemoprevention [published correction appears in Nat Biomed Eng $2020 ; 4$ : 754-5]. Nat Biomed Eng 2018 ; 2 : $27-37$.

28. Dos Santos LI, Galvão-Filho B, de Faria PC, et al. Blockade of CTLA-4 promotes the development of effector $C D 8^{+} T$ lymphocytes and the therapeutic effect of vaccination with an attenuated protozoan expressing NY-ESO-1. Cancer Immunol Immunother 2015 ; 64 : 311-23.

29. Hassan $\mathrm{R}$, Alley $\varepsilon$, Kindler $\mathrm{H}$, et al. Clinical response of live-attenuated, listeria monocytogenes expressing mesothelin (Crs-207) with chemotherapy in patients with malignant pleural mesothelioma. Clin Cancer Res $2019 ; 25$ : 5787-98.

30. Lu RM, Hwang YC, Liu IJ, et al. Development of therapeutic antibodies for the treatment of diseases. J Biomed Sci $2020 ; 27: 1$.

31. Kleinpeter $\mathrm{P}$, Fend $\mathrm{L}$, Thioudellet $\mathrm{C}$, et al. Vectorization in an oncolytic vaccinia virus of an antibody, a Fab and a scFv against programmed cell death-1 (PD-1) allows their intratumoral delivery and an improved tumorgrowth inhibition. Oncoimmunol $2016 ; 5$ : el220467.

32. Chowdhury S, Castro S, Coker C, et al. Programmable bacteria induce durable tumor regression and systemic antitumor immunity. Nat Med 2019; 25 : 1057-63.

33. Wang G, Kang X, Chen KS, et al. An engineered oncolytic virus expressing PD-Ll inhibitors activates tumor neoantigen-specific T cell responses. Nat Commun $2020 ; 11: 1395$.

34. Baeuerle PA, Reinhardt C. Bispecific T-cell engaging antibodies for cancer therapy. Cancer Res $2009 ; 69: 4941-4$.

35. Fajardo CA, Guedan S, Rojas LA, et al. oncolytic adenoviral delivery of an egfr-targeting $T$-cell engager improves antitumor efficacy. Cancer Res $2017 ; 77: 2052-63$.

36. Barlabé $P$, Sostoa J, Fajardo CA, et al. Enhanced antitumor efficacy of an oncolytic adenovirus armed with an EGFR-targeted BiTE using menstrual blood-derived mesenchymal stem cells as carriers. Cancer Gene Ther 2020 ; $27: 383-8$.

37. Guo ZS, Lotze MT, Zhu Z, et al. Bi- and tri-specific T cell engager-armed oncolytic viruses: next-generation cancer immunotherapy. Biomedicine $2020 ; 8: 204$

38. Pol J, Le Bœuf F, Diallo JS. Stratégies génétiques, immunologiques et pharmacologiques au service d'une nouvelle génération de virus anticancéreux. Med Sci (Paris) $2013 ; 29: 165-73$.

39. Catros V, Dessarthe B, Thedrez A, Toutirais 0 . Les récepteurs de nectines/ nectines-like DNAM-1 et CRTAM - Immuno-surveillance ou échappement tumoral ? Med Sci (Paris) $2014 ; 30: 537-43$.

40. Kitten 0 , Martineau P. Les formats alternatifs aux anticorps. Fragments et nouvelles charpentes. Med Sci (Paris) 2019; 35 : 1092-7.
TIRÉS À PART

I. Dimier-Poisson 